\title{
A Model for Coopetition Evolution of Software Industrial Virtual Cluster
}

\author{
Liu Kewen ${ }^{1,2}$ and Gao Changyuan ${ }^{1}$ \\ 1. Harbin University of Science and Technology, \\ School of Management, Harbin, China \\ ${ }^{2}$.Harbin University of Commerce, \\ School of Computer and Information Engineering, Harbin, China \\ avenn@163.com
}

\begin{abstract}
The application of complex network theory can clearly reveal the coopetition activities between inter-members in software industrial virtual cluster. The research result shows that neither simplex cooperation nor competition, but it is a kind of dynamic equilibrium of coopetition. The empirical is analyzed by using the example of cloud computing. In the process of coopetition evolution, the distribution functions of node strength are from random to power law and eventually to random.
\end{abstract}

Keywords: Software Industrial Virtual Cluster (SIVC),coopetition, complex networks, cloud computing

\section{Introduction}

The Harvard University's Professor Adam M.Brandenburger and Yale University's Professor Barry J.Nalebuff are the earliest researchers theoretically delving into the industry cluster from coopetition. They have respectively analyzed the participants in commercial activities as well as their competition and cooperation elements, proposed the opinion on how to cooperate with the participants with rivals involved for jointly creating a greater market. M.Bengtsson and S.Kock have observed that the coopetition may situate within different scopes of value chain [1]. Porter has pointed out that: "cluster enhances both the competition and cooperation. Due to different scopes and participants, the two things may coexist. Again, for this reason, the coopetition relationship between enterprises can offer incentives and avoid excessive competition at the same time"[2].As the economic globalization and informatization boost, the industry cluster has presented the tendency of virtual development in the dimension of space. In other words, after its initial formation, the industry cluster has showed its enlargement in investment amounts in certain regions at a given time and the spatial expansions formed hereof, including overall network capacity, sales volume, market shares, profiting capacity and innovation ability.

Paul Tracey et al declare that, considering enterprises are no longer limited to local business, but find appropriate worldwide production development and marketing partners, thus the innovation networks between enterprises may be increasingly expanded and internationalized. These innovation networks would possess dynamic characteristics same to clusters like Silicon Valley and Route 128. Moreover, by helping with strategies adjustment and rapid reactions to the technologies and markets, they may enable enterprises to obtain technologies, knowledge and resources on a global scale[3]. Therefore, by breaking through the geographical spatial scope of traditional industry cluster, the advantages ever possessed by geographical proximity could be achieved in the virtual space. In this case, the cluster is no longer a closed local system, but an open 
cluster network. The rapidly developed information and communication technology and economic globalization would also provide technical conditions and market opportunities for the development of virtual industry cluster. This paper probed in coopetition activities of virtual cluster in software industry and established a related model as well as analyzed coopetition activities of virtual cluster in software industry and revealed its evolution law through the latest development layout of global software industry led by cloud computing. It plays an important referential role in delving into the coopetition characteristics and healthy development for the virtual cluster in software industry.

\section{Analysis for Coopetition Activities of in Software Industrial Virtual Cluster}

\subsection{Definition of Relevant Concepts}

Despite the rapid development of software industry, there has no consistent definition neither by the domestic and foreign scholars nor the industry. This paper defines software industry as a summation of all economic activities and relations relative to the software products and software services. Such a definition may remain to be further discussed, but the understanding for software industry and its law is kind of useful. The software products usually include the system software (operating system and database management system), support software (middleware, network management software and program development tool) and application software (industry application software and common application software). The software services involve the service contents about the software, mainly including the information system integration, operation and maintenance, data processing, software testing, consultation and evaluation, and training. The enterprises in the global software industry fall broadly into three categories: independent large software enterprises, software department in large IT enterprises as well as small and medium-sized software enterprises.

According to the concept of virtual industrial cluster proposed by Professor Molina from Centre for Integrated Manufacturing Systems(CSIM) at ITESM University in Mexico, the members thus can share the market opportunities and participate in the global business[4,5]. It is a concept equivalent to an aggregation comprised by enterprises with certain expertise, whose main function is to participate in the virtual enterprise operation by providing and adjusting core competence of member enterprises. At the same time, researchers have made numerous detailed studies on the virtual industry cluster from multiple perspectives. Based on these, they widely held that, virtual industry cluster is a novel cluster form generated from the development opportunity that showed by the novel economy characterized by the modern information technology and global economic integration. This kind of cluster form can break through the geographic restrictions of traditional industry cluster[6]. By combining the software industry and definition of virtual industry cluster, this paper believes that the software industrial virtual cluster (SIVC) is a community for software developers, distributors, service providers, users and related organizations to seek global cooperation and competition by adopting the modern information technology.

\subsection{SIVIC Coopetition Activities}

Due to the high technology and innovation characteristic of the software industry, SIVC coopetition activities are not mutually exclusive and manifest the cooperation and competition simultaneously at different development stages of technology and market.

2.2.1. Coopetition Activities in Product Level: As an information product, the software has a relatively strong network effect. Katz and Shapiro call the complementarity of information products as the network effect and divide it into direct network effect and 
indirect network effect[7]. The direct network effect refers to the interdependency between customers using the same product, contributing the utility of consumers to be the increasing function of product consumption scale. It makes the information products possess the required scale economy, if user scale exceeds "critical quantity", the user consumption and utility may be mutually enhanced due to the network effect and the positive feedback mechanism may appear. The indirect network effect is caused by the complementarity in essence, but generated from the technical complementarity between basic products and subsidiary products, such as software and hardware. For these grounds, the complementarity of network economy reflects the necessity of SIVC cooperation and the "lock" is the result of SIVC competition.

2.2.2. Coopetition Activities in Technology Level: The standard plays an important role in the technological development and commercial practice, so it is usually started from the standard competition. There are numerous ways to turn the technology into the standard. A regulative entity or a dominant enterprise with the capability of implementation may sometimes facilitate a market standard. But under the absence of an entity or enterprise facilitating a market to form the compatibility standard, it is spontaneously formed by drastic market competition. The corresponding cooperation alliance would be formed in the process of formulating the standard at the same time, whose objective is to earn profit from formulating compatibility standard to the hilt [8].

2.2.3. Coopetition Activities in Market Level: The high concentration in software industry and obvious scale economies effect are for the economic characteristics of software products. The development of software industry and technology begins in the system software, whose high lock effect enables it to present the feature of oligopolistic market structure in the early stage. For instance, the scholars have found out in their case studies of business that, during the long-term coopetition of SAP and ORACLE, two enterprises leading in the domain of application software, especially in ERP, SAP make its users use the ORACLE database, wherein the latter provides database service for SAP users. SAP and ORACLE are both technology partnership and competitor for ERP products[9]. For this reason, large software enterprises with same market segments would compete with each other, while small and medium-sized software enterprises would cooperate with the large software enterprises. And simultaneously, many software enterprises having complementary goods may cooperate with each other.

2.2.4. Coopetition Activities in Industry Level: The global software industry is changing extensively and profoundly in series, the industrial form is accelerating the reconstruction, the transboundary coopetition between enterprises is becoming even more violent, the novel coopetition pattern between the software and hardware providers is forming, as well as the Android and iOS are presenting the new trend of diversified coopetition in the existing circle of operating system.

It can be seen from the above analysis that, the software industry has a higher dynamic coopetition level and the competitive advantages of software enterprises come from temporary advantages during the coopetition interactive process. Taking the innovation as a main coopetition way, SIVC makes the dynamic coopetition centered with the control and adjustment of the existing standards. The software enterprises establish the market standard by the rapid market expansion and keep the predominance by continuous innovation.

\subsection{Complex Network Characteristics in SIVC Coopetition Activities}

Due to the coopetition strategies of SIVC members and changes in external market demands, SIVC coopetition activities have the complex network characteristics. 
2.3.1. Dynamic Growth: In order to adapt to changes in market conditions and maintain the enterprise competitiveness, SIVC members change constantly. The member enrollment, obsolescence or withdrawal would lead to the recombination of coopetition relationship. Hence, SIVC coopetition activities show a dynamic open and growth.

2.3.2. Network Complexity: If a member participates in two market segmentation fields at the same time in the SIVC coopetition activities, the coopetition of several fields in software industry would form the cross-nested structure and reflect the network complexity.

2.3.3. Community: The cross-nested structure in the transboundary coopetition has formed a community by connecting several synergic SIVC members with the centrality of leading enterprises in each segmentation field. Again, the nodes inside of each community have established the coopetition relationship with nodes in other communities. In this way, not only the coopetition relationship between nodes inside of the community, but also the coopetition relationship between nodes among different communities is then formed.

2.3.4. Preferential Attachment: The obvious positive feedback phenomenon exists in the evolution of SIVC coopetition activities, i.e. those member nodes having stronger strength may obtain more coopetition chances and achieve the connection with more nodes in the complex network, and in reverse, the strength would be rapidly enhanced.

\section{Establishment and Analysis of SIVC Coopetition Model}

\subsection{Establishment of the Model}

The cooperation and competition in the complex network has always been a research hotspot among scholars. By the bipartite network method[10], an analysis tool for complex network, this paper constructed coopetition activities of SIVC members in related fields of the software industry as a weighted bipartite network $H=(M, N, E)$, assumed the collection of all SIVC members as $\mathrm{U},|\mathrm{U}|=\mathrm{M}$, the collection of all coopetition fields as $\mathrm{R},|\mathrm{R}|=\mathrm{N}$, wherein nodes in $\mathrm{R}$ can be operating system, browser software, network security software and other segmentation fields in software industry and $\mathrm{E}$ is the collection of edges between $\mathrm{U}$ and $\mathrm{R}$. An edge was established between the coopetition participants in the same field in software industry, wherein the weight value on which represent the number of projects taking part in the coopetition or coopetition strength between them. The weighted bipartite network is made up of m nodes in $U, n$ nodes in $\mathrm{R}$ and edge e between $\mathrm{U}$ and $\mathrm{R}$, wherein $\mathrm{e}=(\mathrm{i}, \mathrm{j}) \in \mathrm{H},{ }^{w_{i j}}$ represents the weight on the edge[11].

The weighted bipartite network can be represented by a $\mathrm{M} \times \mathrm{N}$ adjacent matrix $\mathrm{W}=\left(\mathcal{w}^{i j}\right)$, wherein $\mathrm{i}=1,2, \ldots, \mathrm{M}$ and $\mathrm{j}=1,2, \ldots, \mathrm{N}$. Each element ${ }^{w_{i j}}$ in the matrix represents the weight of edge $\langle i, j\rangle$.

As the comprehensive representation of local information and self-capability in the complex network, the node degree and adjacent weight reflected by node strength can preferably manifest its important role in the network. The node strength ${ }^{S_{i}}$ of node i in collection $\mathrm{U}$ is defined as:

$$
S i=\sum_{j \in \tau(i)} w_{i j}
$$


This paper used $p(S)$ to represent the distribution function of node strength in $\mathrm{U}$, whose implication is the ratio of the number of nodes with the strength of $S_{U}$ accounted in the total number $n_{U}$ of nodes in U. i.e.:

$$
p(S)=\frac{n\left(S_{U}\right)}{n_{U}}
$$

(2)

Similarly, the node strength $\mathrm{Sk}$ of node $\mathrm{k}$ in $\mathrm{R}$ and the distribution function of node strength are:

$$
S k=\sum_{l \in \tau(k)} w_{k l}
$$

$$
p(J)=\frac{n\left(S_{R}\right)}{n_{R}}
$$

$\mathrm{n}$ is the total number of edges in network $\mathrm{H},{ }^{n(E w)}$ represents the number of edges with the weight of $\mathrm{w}$, then the distribution function of edge weight can be represented as:

$$
p\left(w_{U}\right)=p\left(w_{\mathrm{R}}\right)=\frac{n(E w)}{n}
$$

\subsection{Evolution Process of SIVC Coopetition}

SIVC coopetition activities possess two significant evolution mechanisms the complex network has: topological growth and edge weight evolution. Hence, the construction algorithm is shown below:

Step 1: Initial network. It covers ${ }^{m_{0}}{ }^{n}$ nodes in $\mathrm{U},{ }^{n}{ }^{0}$ nodes in $\mathrm{R}$ and $e^{e}$ edges between $\mathrm{U}$ and $\mathrm{R}$, wherein each edge is given the initial weight $w_{0}$. Assume that each time step would add new nodes of certain quantity in $\mathrm{U}$ and R. Mark the strength of node $\mathrm{i}$ at time $\mathrm{t}$ as $S_{i}(t)$.

Step 2: Topological growth. Add a new node in the collection $\mathrm{R}$ and the newly added node would be connected to $\mathrm{m}$ nodes involved in that time in $\mathrm{U}$ with the preferential probability of $P_{i}$.

$$
P_{i}=\frac{S_{i}(t)}{\sum S_{i}(t)}
$$

(6)

Add a new node to the collection $\mathrm{U}$ and that node would be connected to $\mathrm{n}$ nodes involved in that time in $\mathrm{U}$.

(7)

$$
P_{j}=\frac{S_{j}(t)}{\sum S_{j}(t)}
$$

The preferential attachment mechanism of complex network enlarges the chances of getting selected. In other words, the more active the coopetition of SIVC members is or the higher the market maturity and technical innovation degree is, then the more connections nodes would possess.

Step 3: Dynamic evolution of edge weight. A new edge $\langle\mathrm{n}, \mathrm{i}\rangle$ between the node $\mathrm{i}$ of existing SIVC member and the new node $n$ of software segmentation field is formed at a certain time step, as shown in dotted portion in Figure 1, wherein the newly added edges are given of the edge weight ${ }^{w 0}$. At the same time, as time goes by, the coopetition in the 
network has been accumulated. The weight of edge between the node $\mathrm{i}$ and other connected nodes $\mathrm{k}$ is readjusted:

(8)

$w_{i k} \rightarrow w_{i k}+\Delta w_{i k}$

$$
\Delta w_{i k}=\delta_{i} \frac{w_{i k}}{S_{i}}
$$

Equation (8) and (9) mean that, SIVC adds coopetition activity at a value of $\delta_{i}$ for each time step and the connected edge allocates the newly added coopetition according to the proportion of self-weight $w_{i k}$ in node strength $S_{i}$. Hence, the node strength of user node $\mathrm{i}$ is adjusted as:

$$
S_{i} \rightarrow S_{i}+\delta_{i}+w_{0}
$$

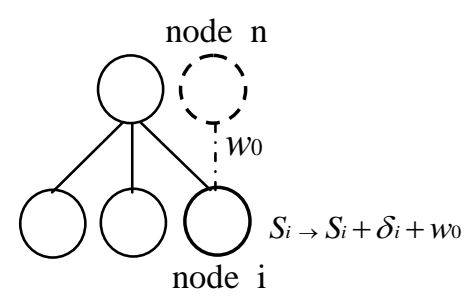

Figure 1.Edge weight evolution of model.

\subsection{Model Analysis}

During the coopetition evolution process of SIVC in a software industry segmentation field, the scale of member nodes in bipartite network is relatively small and coopetition activities are relatively rare. However, as the network scale expands, there appear more and more coopetition activities. As per the mean-field theory[12,13], the distribution on $\mathrm{t}$ by strength $S_{i}$ of ith node in $\mathrm{U}$, if $S_{i}$ is changed continuously, then the change rate of $S_{i}$ is:

$$
\frac{d S_{i}}{d t}=m \frac{S_{i}}{\sum S_{i}}\left(w_{0}+\delta\right)+\sum n \frac{S_{j}}{\sum S_{j}} \delta \frac{w_{i j}}{S_{j}}
$$

The first item on the right of equation (11) represents the situation of node $i$ and its edge after adding a new node in $\mathrm{U}$ and the second item represents the situation of node $\mathrm{i}$ and its edge after adding a new node in $\mathrm{R}$. The total weight adds $w_{0}+\delta_{\text {for }}$ every newly added edge, so the addition of weight for each time step is $(m+n)\left(w_{0}+\delta\right)$

- when $\mathrm{t}=0, \sum s_{i}=\sum s_{j}=e_{0} w_{0}+(m+n)\left(w_{0}+\delta\right) t \approx e_{0} \mathcal{W}_{0}$, put it in the equation (11) and obtain:

$$
\frac{d S_{i}(t)}{d t}=\frac{m w_{0}+m \delta+n w_{0}}{e_{0} w_{0}} S_{i}(t)
$$

Consequently,

$$
S=e^{\frac{m w 0+m \delta+n w 0}{e 0 w 0} t}
$$


When $\mathrm{t}=0$, the bipartite network model is a random model approximately subjected to the exponential distribution. It means that $\mathrm{s}$ small number of SIVC members selected to conduct coopetition activities in their preferential segmentation fields at the initial moments. However, the result is that coopetition activities randomly distributed are formed in few segmentation fields.

when $t$ is large enough, as nodes increase and time goes by, when the network scale is large

enough: $\sum s_{i}=\sum s_{j}=e_{0} w_{0}+(m+n)\left(w_{0}+\delta\right) t \approx(m+n)\left(w_{0}+\delta\right) t$, then put it in the equation (11):

$$
\frac{d S_{i}(t)}{d t}=\frac{(m+n) \delta+m w_{0}}{(m+n)\left(w_{0}+\delta\right)} \frac{S_{i}(t)}{t}
$$

Assume $\alpha=\frac{(m+n) \delta+m w_{0}}{(m+n)\left(w_{0}+\delta\right)}$, then $\frac{d S_{i}(t)}{d t}=\alpha \frac{S_{i}(t)}{t}$. The initial condition of the model is $S_{i}\left(t=t_{i}\right)=m$, then

$$
S_{i}(t)=m\left(\frac{t}{t_{i}}\right)^{\alpha}
$$

The probability for node strength $S_{i}(t)$ of node i less than $\mathrm{S}$ is

$$
P\left(S_{i}(t)<S\right)=P\left(t_{i}>\frac{t}{\left(\frac{S}{m}\right)^{\frac{1}{\alpha}}}\right)=1-P\left(t_{i}<\frac{t}{\left(\frac{S}{m}\right)^{\frac{1}{\alpha}}}\right)
$$

Assume the time for added node $i$ is subject to the uniform distribution, then

$$
P\left(S_{i}(t)<S\right)=1-\frac{t}{\left(\frac{S}{m}\right)^{\frac{1}{\alpha}}} \frac{1}{t+m_{0}}
$$

According to $P(S)=\frac{\partial P\left(S_{i}(t)<S\right)}{\partial S}$, the node strength is subject to the power-law distribution with the exponent of $\beta, P(S) \square S^{-\beta}$, wherein

$$
\beta=2+\frac{n w_{0}}{(m+n) \delta+m w_{0}}
$$

It can be seen from equation (11) that the power exponent is related to $m, n, w_{0}, \delta$ and subject to the power-law distribution law of bipartite network. That is to say, a small number of hotter segmentation markets have centralized massive coopetition activities in SIVC, manifesting power-law distribution characteristic in statistics, wherein the edge weight and node strength have a direct correlation. Other than meeting the power-law distribution, it also presents the heavy-tailed characteristic. Again, with the addition of new nodes and edges, the node degree and edge weight are continuously increasing and when the network is large, the 
increasing tendency of edge weight comes slower and few nodes with larger scale values and edges with larger weights are formed, i.e. key nodes and core enterprises.

There still remains a condition in the bipartite network of this model: as the differentiation of SIVC coopetition boosts, massive nodes are removed along with those connected edges. At the same time, the network scale is reduced, edge number and weights are reduced, and so do coopetition activities.

$$
\frac{d S_{i}(t)}{d t}=\frac{m w_{0}+m \delta+n w_{0}}{e_{0} w_{0}} S_{i}(t)-\frac{(m+n) \delta+m w_{0}}{(m+n)\left(w_{0}+\delta\right)} \frac{S_{i}(t)}{t}
$$

According to the equation (19),

$$
S=t-^{-\frac{(m+n) \delta+m w 0}{(m+n)(w 0+\delta)}} e^{\frac{m w 0+m \delta+n w 0}{e 0 w 0}} t
$$

The first item on the right of equation (19) represents the part of newly added nodes and un-removed edges and the second item represents the part of newly added nodes and removed edges in quantity. In this case, the model is a ruleless and timevarying random network.

\section{The Demonstration of SIVC Coopetition in Cloud Computing Field}

\subsection{The Pattern of SIVC Coopetition in Cloud Computing Field}

The technological development, market demand and other factors have contributed the market scale of cloud computing industry to be enlarged quickly, so the cloud computing market currently faces a complex coopetition relationship. As a leading enterprise in the circle of cloud computing market, Amazon uses the surplus IT infrastructure resources of enterprises to provide remote cloud services, such as the widely applied IaaS (Infrastructure as a Service) cloud computing pattern. Other than its strong search service, Google also offers a reliable and efficient computing service. There are at least five types of competitors actively taking part in the distribution and competition of cloud computing field from the current pattern. They are: internet service providers represented by Google, Amazon; system integration providers represented by IBM, HP; software providers represented by Microsoft, SaleForce.com; equipment manufacturers represented by Cisco, EMC; telecom operators represented by Verizon, AT\&T. Employing the SIVC coopetition model can give reasonable explanations for the coopetition activities in these enterprises.

The service levels of cloud computing: infrastructure level, platform level and software level have the corresponding service patterns: IaaS (infrastructure as a service), PaaS (platform as a service) and SaaS (software as a service). According to the global market share standings of cloud computing[14], the related manufacturers and coopetition bipartite network in the cloud computing field are shown in Figure 2. An increasing number of manufacturers provide cloud computing services at different levels and part of manufacturers provide cloud computing services of equipment, platform and software at the same time. For instance, Amazon, Google and IBM can provide all the cloud computing services of equipment, platform and software. According to the model in this paper, 9 enterprises in Figure 2 can act as nodes in the collection $U$ and the three service levels in the field of cloud computing can act as nodes in the collection R. 


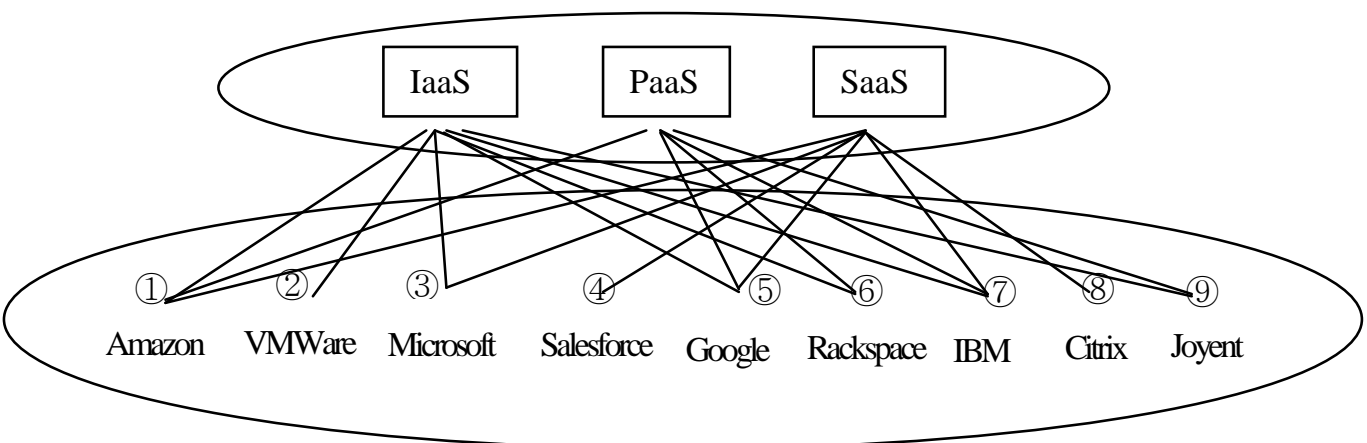

\section{Figure 2. The Coopetition between the 9 Important Members of SIVC in the Cloud Computing Field}

Figure 3 is drawn according to the projection method of bipartite network [17] and Table 1 collects the basic network structural indexes of the weighted network. Table 1 and Figure 3 reflect that the values of $\mathrm{CC}($ Closeness Centrality) at node 1, 3, 5, 7 are all 0.125 in the network. Based on this, Amazon, Microsoft, Google and IBM have participated in the all-round coopetition as key nodes in the field of cloud computing, while other node members may make coopetition in the SIVC-related segmentation fields.

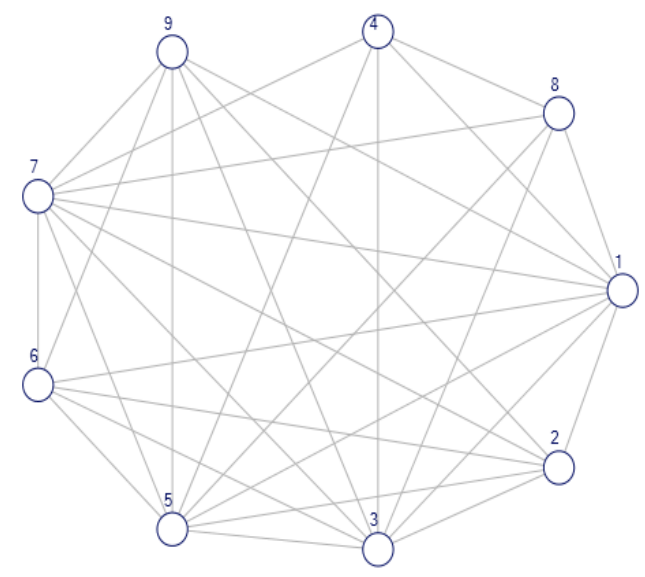

Figure 3. The Topology of Coopetition Networks about the 9 Important Members of SIVC

Table 1. The Basic Statistics of Coopetition Networks about the 9 Important Members of SIVC

\begin{tabular}{c|c|c|l}
\hline Vertex & Degree & $\begin{array}{c}\text { Betweenness } \\
\text { Centrality }\end{array}$ & $\begin{array}{l}\text { Closeness } \\
\text { Centrality }\end{array}$ \\
\hline 1 & 8 & 1.500 & 0.125 \\
\hline 2 & 6 & 0.000 & 0.100 \\
\hline 3 & 8 & 1.500 & 0.125 \\
\hline 5 & 8 & 1.500 & 0.125 \\
\hline 6 & 6 & 0.000 & 0.100 \\
\hline 7 & 8 & 1.500 & 0.125 \\
\hline 9 & 6 & 0.000 & 0.100 \\
\hline 4 & 5 & 0.000 & 0.091 \\
\hline 8 & 5 & 0.000 & 0.091
\end{tabular}




\subsection{Impacts on SIVC Coopetition Exerted by Cloud Computing}

The development of cloud computing exerts an important impact on SIVC coopetition. On one hand, the software industry is engaged in industrial activities centered by development and utilization of the information resources, wherein the priority in software industry has turned from technology-oriented business into service-oriented business. Defining the users' value demand and designing personalized software products based on characteristics of demand by different segmentation market users comes as a key problem and challenge for the development of software manufacturers. On the other hand, the networking character of software impacts the whole behaviors and characteristics of the software market. The pattern of software open-source development fades the life cycle of software products and software providers can update their software online at any time. Therefore, the cloud computing service pattern of socialization, intensification and professionalization uses the reusing and flexible recombination of software to optimize and reconstruct the service process, facilitates the resources aggregation, information sharing and cooperative work between software enterprises and thus forms the serviceoriented computing. These impacts exerted by the cloud computing provide chances and challenges to enhance the coopetition between SIVC software enterprises. Thus, enterprises would takeover frequently and be committed to continuous innovation.

If the scale economy and scope economy in the software industry are weakened by the added market uncertainties and technical changes, the vertical and horizontal nonintegration and decentralization of production in the industry could better face the variable demands and adapt to the market changes. In this way, the coopetition between enterprises in SIVC becomes as the key point. Again, the virtual decentralism and aggregation behaviors of cluster contribute to the coopetition pattern peculiar to the software industry. For this ground, many enterprises have carried out the coopetition interaction with multipoint attachment in several market segmentation fields, formed numerous subgroups in SIVC, and brought about the effect of reduced competition strength and increased profit rate, benefiting the global stability of SIVC system. As the cloud computing develops, participating enterprises achieve the continuous prosperity of the system by conversing and improving the self effectiveness between different SIVC groups, complex network formed by SIVC coopetition and dynamic changes of node strength and distribution conditions, wherein the coopetition pattern has presented a development and evolvement law similar to the ecological system.

\section{Conclusions}

As stated above, an innovation environment is formed based on the high interaction of enterprises in SIVC. As the main content of interaction, the coopetition is a complex dynamic equilibrium, whose essence is to take the coexistence of cooperation and competition as the mutual condition. Again, the coopetition of participants in levels like software products, technology and market is carried out on condition of pursuing efficiency and innovation. In this way, it not only avoids cutthroat competition, but also overcomes the instability in cooperation. According to the SIVC members, software industry segmentation fields, network topological growth and other characteristics, this paper has established the bipartite network evolution model through giving SIVC coopetition relationship weight values and following the principle of preferential attachment. The researches show that, as the SIVC coopetition activities change, the model has experienced the transition from the exponentially distributed random model to the power-law distributed scale-free model and eventually to the random model to certain extent. The evolution of SIVC coopetition shows the continuous innovative development of software industry and participation dynamics of member enterprises, manifesting on the difference of the node strength and edge weight in the weighted bipartite network of various magnitude, which is also an inescapable consequence of the coopetition evolution. 


\section{References}

[1] M.Bengtsson and S.Kock. "Cooperation and Competition in Relationships Between Competitors in Business Networks". Journal of Business \& Industrial Marketing.vol. 14, no. 3, (1999), pp.178-194.

[2] M. E. Porter. "Clusters and the New Economics of Competition". Harvard Business Review.vol. 76, no. 6, (1998), pp.36-38

[3] P. Tracey and G. L. Clark.Alliances, "Networks and Competitive Strategy:Rethinking Clusters of Innovation". Growth and Change. vol. 34, no. 1, (2003), pp.1-16.

[4] A. Molina, C. F. Bremer and W. Eversheim, "Achieving critical mass: a global research network in systems engineering[J]”. Foresight. vol. 3, no. 1, (2001). pp.59-65.

[5] D.Caballero, A.Molina and T.Bauernhansl. "A Methodology to Evaluate Enterprises to Become Members of Virtual Industry Clusters". IFIP - the International Federation for Information Processing.56, pp.443-454(2001).

[6] G. Passiante and G.Secundo. "From geographical innovation clusters towards virtual innovation clusters: the innovation virtual system" the 42nd ERSA Congress, University of Dortmund, (2002) August;Germany, pp. 1-22.

[7] M. Katz and C. Shapiro, "Network Externalities,Competition and Compatibility. American Economic Review". vol. 75, no. 3, (1985), pp.424-440.

[8] R. Axelrod, W.Mitchell, R.E.Thomas,D.S.Bennett, E.Bruderer, "Coalition Formation in Standardsetting Alliances". Management Science.vol. 41, no. 9, (1995), pp.1493-1508 .

[9] E. M. Troesch and P. F. Schikora. "Co-opetition Between SAP and Oracle:The Effects of the Partnership and Competition on the Companies'Success". Journal of Business Case Studies. vol. 6, no. 4, (2010). pp.59-65

[10] R.Lambiotte,M.Ausloos. "N-Body Decomposition of Bipartite Author Networks". Physical Review E. vol. 72, no. 6, (2005).

[11] M.E.Newman, “Analysis of Weighted Networks”. Physical Review E.vol. 70, no. 5, (2004).

[12] A.L.Barabási,R.Albert and H.Jeong. "Mean-field theory for scale-free random networks". Physica A: Statistical Mechanics and Its Applications. vol. 272, no. 1, (1999) . pp.173-187.

[13] S.H.Yook,H.Jeong,A.L.Barabási,Y.Tu. "Weighted evolving networks". Physical Review Letters. vol. 86, no. 18, (2001).pp.5835-5838.

[14] J Bort., "The 10 Most Important Companies in Cloud Computing. Business Insider", Apr 25(2012).

\section{Authors}

LIU Kewen, is a doctoral student in Management Science and Engineering at School of Management, Harbin University of Science and Technology. She is also a vice professor in School of Computer and Information Engineering at Harbin University of Commerce. Her research interests are in the areas of e-commerce, management information system and coopetition.

GAO Changyuan, is a doctoral tutor in Management Science and Engineering at School of Management, Harbin University of Science and Technology. His main research area is High-tech Virtual Industrial Cluster.

This work was financially supported by project of National Natural Science Foundation of China (71072085). 
International Journal of Grid and Distributed Computing Vol. 9, No. 3 (2016) 